\title{
The Formation of Massive Stars
}

\author{
Ian A. Bonnell ${ }^{1}$ and Rowan J Smith ${ }^{2}$ \\ ${ }^{1}$ SUPA, School of Physics \& Astronomy, Universiy of St Andrews, North Haugh, St Andrews, \\ Fife KY16 9SS, UK \\ email: iab1@st-andrews.ac.uk \\ ${ }^{2}$ Institut fuer Theoretische Astrophysik, Albert-Ueberle-Str. 2, 69120, Heidelberg, Germany
}

\begin{abstract}
There has been considerable progress in our understanding of how massive stars form but still much confusion as to why they form. Recent work from several sources has shown that the formation of massive stars through disc accretion, possibly aided by gravitational and Rayleigh-Taylor instabilities is a viable mechanism. Stellar mergers, on the other hand, are unlikely to occur in any but the most massive clusters and hence should not be a primary avenue for massive star formation. In contrast to this success, we are still uncertain as to how the mass that forms a massive star is accumulated. there are two possible mechanisms including the collapse of massive prestellar cores and competitive accretion in clusters. At present, there are theoretical and observational question marks as to the existence of high-mass prestellar cores. theoretically, such objects should fragment before they can attain a relaxed, centrally condensed and high-mass state necessary to form massive stars. Numerical simulations including cluster formation, feedback and magnetic fields have not found such objects but instead point to the continued accretion in a cluster potential as the primary mechanism to form high-mass stars. Feedback and magnetic fields act to slow the star formation process and will reduce the efficiencies from a purely dynamical collapse but otherwise appear to not significantly alter the process.
\end{abstract}

\section{Introduction}

Understanding how massive stars form is important as high-mass stars dominate the luminous, kinematic and chemical output of stars, and thus the evolution of galaxies. Massive star formation is problematic as high-mass stars are rare, and generally form in dense stellar clusters. They rarely if ever form in isolation (De Wit et al. 2005) and need to be understood in the context of forming the full mass distribution of stars. In addition, massive stars are commonly found in close binary systems with other high-mass stars.

There are several outstanding issues that need to be addressed in order to build a complete theory of massive star formation (Zinnecker \& Yorke 2007). Firstly, there is the issue of their actual formation and whether they form from disc accretion as low mass stars do, or from something more exotic such as stellar mergers. Secondly, there is the question as to what drives high-mass stars to form and at what stage is the mass gathered. This argument involves the possibility that the mass is gathered into a single high-mass but prestellar core that collapses into one stellar system, versus the idea that the mass is gathered during the star formation process due to the combined gravitational potential of the stellar cluster in which the proto-massive star resides. Lastly, we would like to understand the properties of massive stars and how they affect their environment, including any subsequent star formation. 


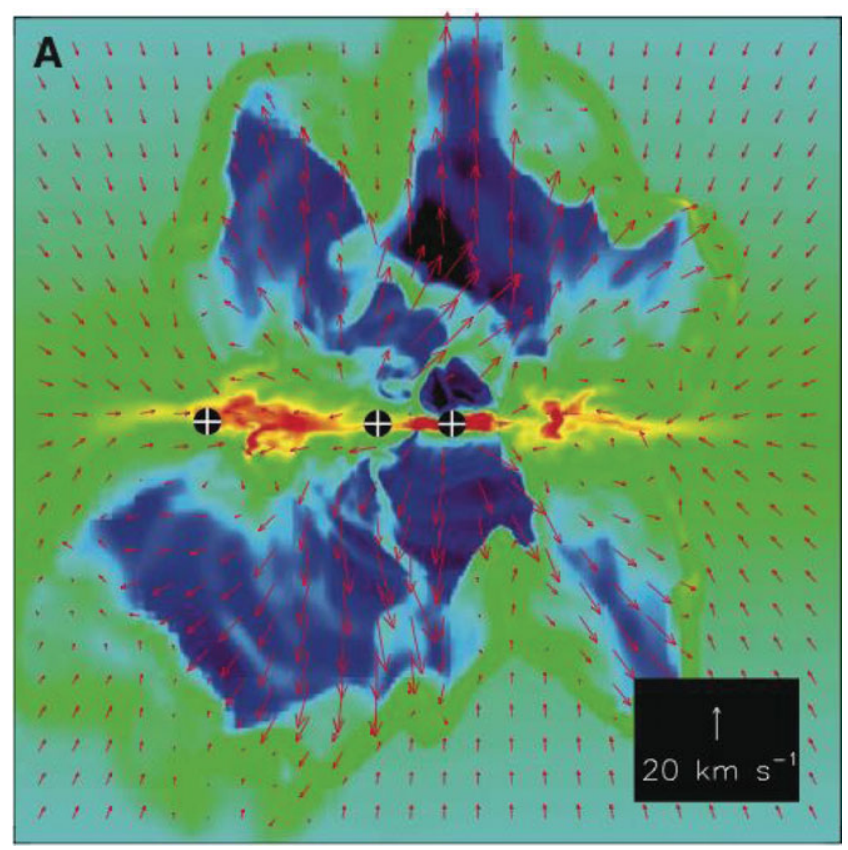

Figure 1. The onset of Rayleigh-Taylor instabilities is shown in a numerical simulation of accretion onto a forming high-mass star. The system is shown edge-on. Note the presence of the low-density bubbles (dark blue) and high density gas (light yellow/red) as well as the arrowed gas velocities (Krumholz et al. 2009).

\section{Disc Accretion Versus Stellar Mergers}

An long-standing problem of massive star formation has been how to circumvent the high radiation pressure from stars with masses greater than 10 solar masses (Khan 1974; Wolfire \& Casinelli 1986 ). Such stars have radiation pressures that can act sufficiently strongly on the dust to reverse the infall, and thus potentially halt mass accretion, limiting the growth of the massive star. Suggestions put forward to circumvent this problem include disc accretion, dust destruction in the inflow (Keto 2003), beaming of the radiation, Raleigh-Taylor instabilities and stellar mergers (Bonnell et al. 1998). Several studies (Yorke \& Sonnhalter 2002; Krumholz et al. 2009; Kuiper et al. 2010) have shown that when increasingly realistic physics is included in models, such as frequency dependent treatment of the radiation, gravitational instabilities in the disc and Rayleigh-Taylor instabilities, the formation of a high-mass star can still occur through disc accretion.

Krumholz et al. (2009) performed the first 3-D simulation including radiation pressure in the flux-limited diffusion approximation, and showed that the discs that formed were unstable and fragmented to form multiple objects. This moved the source of the radiation pressure away from the centre of mass which aided further accretion. The accretion disc itself was fed by a combination of flow around the radiation-driven bubble and RayleighTaylor instabilities inside from the heavier gas on top of the bubbles (Fig.1).

In addition to the successes of disc accretion in forming high-mass stars, it is also increasingly clear that stellar mergers are unlikely to play a role in the formation of all high-mass stars. This is due to the difficulty in obtaining the necessary physical conditions for stellar mergers to occur on timescales of less than a million years (Bonnell et al. 1998; Bonnell \& Bate 2002; Davies et al. 2006). While accretion onto a clusters stellar core can drive the core into collapse and dramatically increase the stellar density as $n \propto m_{*}^{9}$ 
(Bonnell \& Bate 2002), the core itself may dissolve through its gravitational interactions if it becomes uncoupled from the rest of the cluster (Clarke \& Bonnell 2009). This occurs as the core is then a small-N system which has a relatively short evaporation timescale due to two-body interactions in which a central binary ejects the other members. To counteract this, the core must be well coupled to the rest of the cluster such that the excess kinetic energy given to individual objects is smoothly transferred to the outer regions of the cluster where the energy can be shared by many stars. This requires a large cluster with a very high central density that smoothly decreases with radius until scales where the relaxation timescale of the cluster is long.

Recent work (Moeckel \& Clarke 2010) has indeed shown that a large-N cluster of order 30,000 or more is required for accretion to be able to drive the core to such high stellar densities for collisions to occur. However, mergers amongst binary systems are still a possibility even in smaller-N systems, as close binaries are common and their cross section to encounter other stars is much larger (Bonnell \& Bate 2005).

\section{Monolithic Massive star Formation}

One of the leading models for massive star formation invokes the collapse of a single, high-mass prestellar core to directly form a single high-mass star (McKee \& Tan 2003; Banerjee \& Pudritz 2007). The appeal in this model arises from it being a scaled-up version of low-mass star formation where stars are observed forming within low mass gas cores. A further attraction is that observations show that the core mass function (CMF) resembles the stellar IMF (Motte et al. 1998; Testi \& Sargent; Johstone et al. 2000), which has lead some to propose a one-to-one correlation between the two, albeit with some efficiency factor (Alves et al. 2007; Simpson et al. 2008). In order for this model to work, the core must be a completely distinct element of the molecular cloud and must not gain significant mass or fragment during the star formation process.

Smith et al. (2009a) have investigated the relation between the CMF and the stellar IMF through numerical simulations. They find that although the CMF does resemble the IMF, the core masses do not map directly into stellar system masses. This arises due to a combination of geometrical effects during core collapse, and varying amounts of subsequent accretion from the surrounding environment which adds significant dispersion to the relation between the core mass and the final stellar mass. This is not very surprising given that the cores in a clustered region are somewhat artificial in that they do not have distinct boundaries but are instead the high-density peaks of a larger mass distribution (Smith et al. 2008, Kainulainen et al. 2009).

The question of a massive core's fragmentation is one that has been addressed by several studies (Dobbs et al. 2005; Krumholz et al. 2006, 2010). The symetrical, centrally condensed nature of the core, believed to arise if its pre-collapse evolution is quasi-static, helps suppress any fragmentation (eg. Boss 1993?). The difficulty arises in that the core needs to be turbulently supported in order that the equivalent turbulent Jeans mass is of order the core mass. McKee \& Tan modelled this turbulence as an equivalent isotropic sound speed but turbulence is much more complicated and does not support objects isotropically. Instead such objects are rapidly deformed by their internal motions providing the necessary seeds for fragmentation (Dobbs et al. 2005). Even centrally condensed cores that are turbulently supported fragment provided that they are able to remain nearly isothermal (Dobbs 'et al. 2005; Krumholz et al. 2006; 2010).

One solution to this problem that has been suggested is the radiative heating from forming stars internal to the cloud (Krumholz refs). This will work on relatively small scales as the temperature due to radiative heating scales as the distance from the source 
as $t \propto r^{-0.4-0.5}$ (Chakrabarti \& McKee 2005). It also requires that the central sources are already present in the core without having previously affected its density distribution in any way. This is likely to be problematic as the core itself must form quasi-statically, over many dynamical timescales if it is sufficiently centrally condensed. The problem aries in that the core should be highly susceptible to fragmentation on a dynamical timescale during this process, hence before any internal sources could have formed.

It is worth noting here that numerical simulations of larger-scale cluster formation, including feedback and magnetic fields, have not been able to form a massive prestellar core. Instead the accumulating gas fragments before it can be assembled into one object inducing the formation of a stellar cluster and subsequent accretion-driven formation of high-mass stars (see below).

\subsection{Critical Surface Density for Massive Star Formation}

Based on the above arguments to suppress fragmentation, Krumholz \& McKee 2008 have suggested that there is a critical surface density in order to form high-mass stars. This arises due to the short-distances over which radiative heating is effective which then necessitate high gas densities and hence surface densities. Regardless that this may not be sufficient to suppress fragmentation which would occur during the formation phase of the core, there is a simpler explanation for a critical surface density to form high-mass stars. High-mass stars are not formed in isolation, but in stellar clusters that follow a standard IMF. This necessitates a total mass of order $1000 \mathrm{M}_{\odot}$. Given a mean stellar mass of $\approx 0.5 \mathrm{M}_{\odot}$, and assigning a gas density to produce a similar Jeans mass, the system has a size around $1 \mathrm{pc}$ and hence a surface density of order $1 \mathrm{~g} \mathrm{~cm}^{-2}$. Not surprisingly, such a system has roughly the same properties as the ONC would have during the prestellar stage.

\section{Competitive Accretion}

The alternative to monolithic collapse is accretion from a larger-scale reservoir onto originally lower-mass cores to form massive stars. In an embedded stellar cluster containing a significant fraction of the total mass in gas, individual stars can accrete from this reservoir but their individual accretion rates depend on their masses, velocities, gas densities and the tidal fields from the other cluster members (Zinnecker 1982; Bonnell et al. 1997, 2001; Bonnell \& Bate 2006).

Numerical simulations show that molecular clouds fragment down to their thermal or magnetic Jeans masses (Bonnell et al. 1991; Klessen et al. 1998; Klessen 2001; Bate et al. 2003; Jappsen et al. 2005; Bonnell et al. 2006; Federrath et al. 2010). As massive stars form in stellar clusters where the mean stellar mass is of order $0.5 M_{\odot}$, the fragmentation mass must be of that order. Fragmentation is also highly inefficient in that only a small fraction of the mass is initially in the high density fragments (Klessen et al. 1998; Bonnell et al. 2003; Smith et al. 2009b). Accretion from this large-scale reservoir is then a plausible way to form high-mass stars.

The formation of a stellar cluster from a massive clump of molecular gas commences as as soon as the clump is formed and is gravitationally bound (Smith et al. 2009b; Bonnell et al. 2010). The internal turbulence fragments the clump into Jeans mass objects which form into small-N subclusters. The overall collapse drives gas and stars together to form a large- $\mathrm{N}$ cluster. The gravitational potential of the cluster ensures that gas is funneled down to the centre to be accreted by what will become the most massive star. The combination of the higher gas density, and subsequently the star's higher mass ensures 


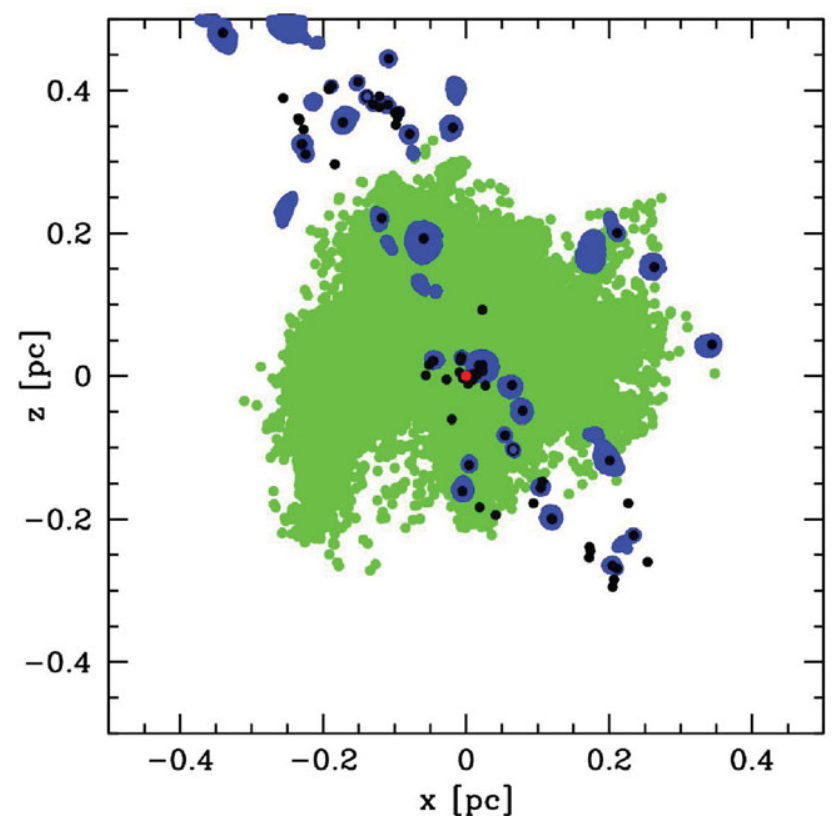

Figure 2. The formation of a stellar cluster from a fragmenting filament. The green dots show the positions of gas which will eventually be accreted by the massive sink (red dot). Black dots show the position of protostars and blue dots show the location of material in bound cores. The gas which will be accreted by the massive protostar is well distributed throughout the clump, but is funneled to the central star by large scale collapse motions (Smith et al. 2009b).

that the central object has a high accretion rate and thus becomes a massive star (Fig. 2). This also helps establish an initial mass segregation.

Smith et al. (2009b, see also Bonnell et al. 2004) show how this results in the simultaneous formation of a massive star and the stellar cluster. The causal relation between the two is that the overall gravitational potential is necessary to gather the mass required for the high accretion rate and thus the formation of the high-mass stars. Forming protoclusters therefore start off as highly fragmented, elongated dispersed objects and evolve towards more well defined centrally condensed spherical objects dominated by the central source. In fact, synthetic maps predict that they will appear less fragmented in continuum maps once fragmentation has occurred and the central temperatures increase due to radiative heating (Smith et al. 2009b).

\section{Feedback from Massive Stars}

Smith et al. (2009b) included an overestimate of radiative heating from protostars and did not find any massive prestellar core. The radiative heating does result in a lower fragmentation rate suppressing some of the low-mass stars from forming but cannot halt the ongoing accretion from large scales as the warm gas remains bound to the cluster. Similarly, Urban et al. 2010 considered the effect of dust heating on a cluster that was prone to fragmentation, heating reduced fragmentation and increased the characteristic stellar mass.

Dale et al. (2005) included ionisation from a central O star and found the overall cluster dynamics largely unaffected as the ionisation found the weakest point in the surrounding cloud through which to escape. This produced a one-sided HII region but 
did not affect the fragmentation or accretion. Including stellar winds in a forming stellar cluster, Dale \& Bonnell (2008) showed that the cloud is partially supported and has a slightly reduced rate of star formation. Nevertheless, the dynamics of the accretion were largely unaffected and no massive prestellar cores were formed. Peters et al. (2010) also found that the development of an HII region is not in itself sufficient to stop accretion. It is generally difficult to stop accretion onto massive stars via feedback as it occurs primarily along a few dense well shielded filaments (Smith et al. 2010; Wang et al. 2010).

A recent result from Wang et al. (2010) included both outflows from stars and magnetic fields in an attempt to support the forming cloud. Similar to the Dale \& Bonnell result, they found that the outflows helped support the cloud reducing the star formation rate but did not halt the accretion from the shared reservoir that ultimately forms the massive star.

In all numerical studies to date, there has been no occurrence of a massive prestellar core being formed that will collapse directly into a massive star. The reason for this lies in the large scale potential in which massive stars form. Once a stellar cluster or similarly bound gas core is formed, there exists a well defined gravitational potential. Gas falling into this potential can either enter as low density dispersed gas or as highdensity, gravitationally bound fragments. In the latter case, the fragments collapse rapidly on their own dynamical timescale to form low-mass stars. In the former case, the gas is tidally unbound and will fall into the potential seeking out a potential minimum defined by an existing object. The accretion of this infalling gas onto the young stars is the competitive accretion process. We cannot envision a way that gas infalling into a well defined potential can form into a gravitationally bound core containing multiple Jeans masses without either being accreted by the stars present in the cluster or fragmenting into low-mass stars (Smith et al. 2009b). To date, no feedback processes, even in the presence of magnetic fields (Wang et al. 2010), have been found which alter this.

\section{Binary systems}

It has long been realised that most high-mass stars are members of binary systems and that these systems often comprise two massive components in a tight orbit of $<1$ AU. How such systems form is unclear. We expect that massive stars should commonly be in binary systems either through three body capture in the cores of dense clusters or through a disc fragmentation process (Kratner Metzner 2006; Krumholz et al. 2009). In both cases, the expectant binary system will still be fairly wide of many tens to $100 \mathrm{AU}$. Hardening such systems is possible through accretion (Bonnell \& Bate 2005) or two-body relaxation in clusters (Heggie 1975) although at present it is not certain that any posited method can actually form the closest systems.

\section{Conclusions}

Massive star formation is now believed to proceed via disc accretion. Stellar mergers may play a minor role in close binary systems or very massive stellar clusters but cannot be the primary mechanism to form massive stars. Radiation pressure does not appear to halt accretion due to to combination of accretion through a disc, and possibly gravitational instabilities in the disc and Rayleigh-Taylor instabilities in the radiation pressure driven bubbles.

Massive prestellar cores will form massive stars, but the question is whether such objects ever exist. Theoretical expectations and numerical simulations both indicate that such cores are unlikely to form without first fragmenting or accreting onto pre-eisting 
stars. In contrast, clustered accretion appears to be a viable mechanism to form massive stars, unimpeded by either magnetic fields or feedback. It does require that massive stars form in stellar clusters and cannot explain the formation of isolated massive stars without the necessity of a high Jeans mass. To date, simulations of feedback have found that it has a limited effect on the massive star formation, but it does slow the large-scale star formation and reduces the star formation efficiency.

\section{References}

Alves J., Lombardi M., Lada C. J., 2007, A\&A, 462, L17

André P., Belloche A., Motte F., \& Peretto N., 2007, A\&A, 472, 519

Banerjee R. \& Pudritz, R., 2007, MNRAS, 660, 479

Bate M. R., Bonnell I. A., \& Bromm V., 2003, MNRAS, 339, 577

Bonnell, I. A. \& Bate, M. R., 2005, MNRAS, 362, 915

Bonnell, I. A. \& Bate, M. R., 2006, MNRAS, 370, 488

Bonnell, I. A., Bate, M. R., Clarke, C. J., \& Pringle, J. E., 2001a, MNRAS, 323, 785

Bonnell I. A., Bate M. R., \& Vine S. G., 2003, MNRAS, 343, 413

Bonnell I. A., Bate M. R., \& Zinnecker H., 1998, MNRAS, 298, 93

Bonnell I. A., Clarke C. J., Bate M. R., \& Pringle J. E., 2001, MNRAS, 324, 573.

Bonnell I. A., Clarke C. J., \& Bate M. R., 2006, MNRAS, 368, 1296

Bonnell I., Martel H., Bastien P., Arcoragi J.-P., \& Benz W., 1991, ApJ, 377, 553

Bonnell I. A., Smith R. J., Clark P. C., \& Bate M. R., 2010, MNRAS, in press

Chakrabarti S. \& McKee C. F., 2005, ApJ, 631, 792

Dale, J. E. \& Bonnell, I. A. 2008, MNRAS, 391, 2

Dale, J. E., Bonnell, I. A., Clarke, C. J., \& Bate, M. R. 2005, MNRAS, 358, 291

de Wit W. J., Testi L., Palla F., \& Zinnecker H., 2005, A\&A, 437, 247

Dobbs C. L., Bonnell I. A., \& Clark P. C., 2005, MNRAS, 360, 2

Federrath C., Banerjee R., Clark P. C., \& Klessen R. S., 2010, ApJ, 713, 269

Heggie, D., 1987, MNRAS, 173, 729

Jappsen A.-K., Klessen R. S., Larson R. B., Li Y., \& Mac Low M.-M., 2005, A\&A, 435, 611

Kahn, F. D. 1974. A\&A 37, 149.

Kainulainen J., Lada C., Rathborne J. M., \& Alves J F., 2009, A\&A, 497, 399

Keto, E., 2003, ApJ 580, 980986.

Klessen R. S., 2001, ApJ, 556, 837

Klessen R. S., Burkert A., \& Bate M. R., 1998, ApJ, 501, L205

Kratter, K. \& Matzner, C., 2006, MNRAS, 373, 1563

Krumholz M., Klein R. I., \& McKee C. F., 2005, ApJ, 618, L33

Krumholz M. R., Klein R. I., McKee C. F., Offner S. S. R., \& Cunningham A. J., 2009, Sci, 323,754

Krumholz M. R. \& McKee C. F., 2008, Natur, 451, 1082

Larson R. B., 2005, MNRAS, 359, 211

McKee, C.F. \& Tan, J.C., 2003, ApJ, 585, 850

Motte F., Andre P. \& Neri R., 1998, A\&A, 336, 150

Nutter D. \& Ward-Thompson D., 2007, MNRAS, 374, 1413

Peters T., Banerjee R., Klessen R. S., Mac Low M.-M., Galván-Madrid R., \& Keto E. R., 2010, ApJ, 711, 1017

Simpson, R.J., Nutter D., \& Ward-Thompson D., 2008, MNRAS, 391, 205

Smith R. J., Clark P. C., \& Bonnell I. A., 2008, MNRAS, 391, 1091

Smith R. J., Clark P. C., \& Bonnell I. A., 2009, MNRAS, 396, 830

Smith R. J., Longmore, S., \& Bonnell I. A., 2009, MNRAS, 400, 1775

Testi L., Sargent A., 1998; ApJL, 508, L91

Urban A., Martel, H., \& Evans N. J., 2010, ApJ, 710, 1343

Wang P., Li Z.-Y., Abel T., \& Nakamura F., 2010, ApJ, 709, 27 
Weidner C. \& Kroupa P., 2006, MNRAS, 365, 1333

Weidner C., Kroupa P., \& Bonnell I. A. D., 2010, MNRAS, 401, 275

Wolfire, M. G. \& Cassinelli, J. P. 1987. ApJ 319, 850867.

Yorke, H. W. \& Sonnhalter, C. 2002, ApJ 569, 846.

Zinnecker H., 1982, New York Acad. Sci. Ann., 395, 226

Zinnecker H., Yorke, H., 2007, ARA\&A, 45, 481 